\title{
The Effects of Medical or Psychiatric Diseases on the Association between Morningness-Eveningness and Somatization
}

\author{
Bum Joon Seok ${ }^{1}$, Sehyun Jeon', Seong-Jin Cho², and Seog Ju Kim ${ }^{1,3}$ \\ ${ }^{1}$ Department of Psychiatry, Samsung Medical Center, Seoul, Korea \\ ${ }^{2}$ Department of Psychiatry, Gachon University Gil Medical Center, Incheon, Korea \\ ${ }^{3}$ Department of Psychiatry, Sungkyunkwan University School of Medicine, Seoul, Korea
}

\begin{abstract}
Objective: Morningness-eveningness (M-E) has been found to be associated with various psychiatric disorders or symptoms. However, the relationship between $\mathrm{M}-\mathrm{E}$ and somatization has not yet been fully investigated. This study aims to explore the relationship between M-E and somatization. Methods: A total of 171 subjects (mean age: $41.1 \pm 8.2$ years; $42.7 \%$ males) were recruited from the Incheon area, Republic of Korea. All subjects were asked to complete questionnaires including the Horne and Ostberg morningness-eveningness questionnaire (MEQ), the Center for Epidemiological Studies Depression Scale (CES-D), and the Symptom Checklist Somatic from the Symptom Checklist-90-Revised (SCL-SOM). Multiple regression analysis was used to test hypotheses. Results: The MEQ and CES-D scores were predictors for the SCLSOM score (MEQ: $\beta=-0.185, p=0.001$; CES-D: $\beta=0.234, p<0.001$ ). In subjects without medical diseases, both the MEQ and CES-D were predictors for the SCL-SOM score (MEQ: $\beta=-0.179, p=0.007$; CES-D: $\beta=0.220, p<0.001$ ). However, only the CES-D score, and not the MEQ score, was a predictor in subjects with medical diseases (CES-D: $\beta=0.230, p=0.019$ ). Similarly, the CES-D score was the only significant predictor in subjects with psychiatric diseases (CES-D: $\beta=0.277, p<0.001$ ); both the MEQ and CES-D scores were significant predictors in subjects without psychiatric diseases (MEQ: $\beta=-0.243, p<0.001$; CES-D: $\beta=0.203, p=0.002$ ). Conclusion: M-E and somatization are significantly associated, independent of depression or anxiety status, indicating that somatization is independently influenced by morning-evening preference. An association between M-E and somatization is evident only in subjects without medical or psychiatric problems.
\end{abstract}

Key Words: Chronology; Circadian rhythm somatic symptom; Depression

Received: December 28, 2018 Accepted: January 21, 2019

Corresponding author: Seog Ju Kim, MD, PhD, Department of Psychiatry, Sungkyunkwan University School of Medicine, Samsung Medical Center, 81 Irwon-ro, Gangnam-gu, Seoul 06351, Korea.

Tel: 82-2-3410-3583, Fax: 82-2-3410-0050, E-mail: psychical@hanmail.net

(a) This is an Open Access article distributed under the terms of the Creative Commons Attribution Non-Commercial License (https://creativecommons.org/licenses/bync/4.0) which permits unrestricted non-commercial use, distribution, and reproduction in any medium, provided the original work is properly cited.

\section{INTRODUCTION}

Somatization refers to psychological discomfort associated with unexplained physical symptoms. Somatization is understood as the conversion of emotional distress into somatic symptoms to focus attention on physical issues rather than intolerable psychological anxiety. Somatization is often evident in patients with mood or anxiety disorders [1].

The morning/evening preference in terms of mental or physical activity or sleep defines morningness-eveningness (M-E). Circadian preference varies individually. Morning-type people operate best in the morning and readily tire in the evening [2]. M-E has been reported to be associated with several psychiatric conditions. Those who prefer evenings exhibit more depressive symptoms [3-6]. Evening-type people were also reported to exhibit more behavioral problems [7], addiction problems [8], and adulthood attention deficit hyperactivity disorder (ADHD) [9]. M-E is associated with not only psychiatric disorders, but also certain personality traits [10-14] and mental and physical premenstrual symptoms $[15,16]$.

Here, we explore the relationship between M-E and somatization. To the best of our knowledge, only a few studies have addressed this association to date. A recent study found that both somatization and M-E were strongly associated with depression, 
but somatization and M-E were not strongly linked [17]. In the cited study, a short (40-item) version of the Sleep Wake Pattern Assessment Questionnaire (SWPAQ) was used to assess M-E. It may be different from the situation in Korea due to the difference of culture and geographic factors.

If $\mathrm{M}-\mathrm{E}$ and somatization were in fact associated, it would be necessary to determine whether the association was independent or influenced by depression or anxiety. Also, the effects of age and gender should be explored, as these factors affect M-E $[18,19]$. The relationship between $\mathrm{M}-\mathrm{E}$ and medical or psychiatric illness would also merit attention [20,21]. Here, we explored the relationship between M-E and somatization, and factors affecting that association. We hypothesized that 1) M-E and somatization are significantly associated, independent of depression or anxiety, and 2 ) this association varies by gender, age, and medical/psychiatric disease status.

\section{METHODS}

\section{Subjects}

Adults ( $\geq 19$ years of age) were recruited from hospitals, apartments, churches, and public health centers in the Incheon area, Republic of Korea, via posters and brochures. Demographic variables such as age, gender, and medical disease status were obtained from self-reports. The presence/absence of Axis I psychiatric disorders was identified by psychiatrists during an interview, based on the Diagnostic and Statistical Manual of Mental Disorders-IV-TR (DSM-IV-TR).

A total of 171 subjects were recruited, 73 of whom were male and 98 female, with mean age $41.1 \pm 8.2$ years (the mean ages of males and females did not differ significantly). The study protocol was approved by the Institutional Review Board of Gachon University of Medicine and Science, and all subjects provided written informed consent.

\section{Instruments}

We used the Korean version of the Horne and Ostberg M-E questionnaire (MEQ) to measure morning-evening preference. The MEQ features 19 mixed-format questions. A higher MEQ score reflects greater morningness [2]. The Korean version of the MEQ was translated from the original MEQ and internal consistency was verified [22].

The Korean version of the Symptom Checklist Somatic (SCLSOM), derived from the Symptom Checklist-90-Revised (SCL90-SOM), was used to measure somatization [23,24]. The SCL-90SOM consists of 90 questions measuring somatization, interpersonal sensitivity, depression, anxiety, hostility, fear, paranoia, and psychological disease. Of these, 12 items explore physical discomfort.

The Korean version of the Center for Epidemiological Studies Depression Scale (CES-D) was used to measure depressive symptoms. High CES-D scores indicate high levels of depressive symptoms [25]. The Korean version of the CES-D is well verified but tends to score higher than the original version [26].
To measure current anxiety, the Korean version of the StateTrait Anxiety Inventory (STAI) was employed [27]. The STAI features subscales assessing anxiety state (the current anxiety level) and anxiety traits (congenital emotional characteristics). We used the state anxiety subscale (STAI-S) because current anxiety can affect somatization.

\section{Statistical analysis}

SPSS Statistics ver. 24 (IBM Corp., Armonk, NY, USA) was used for all statistical evaluations. In terms of demographic variables, the independent t-test was employed to compare continuous data. We used the chi-squared test to compare categorical demographic variables. Pearson correlations were calculated and stepwise multiple regression analyses performed to statistically test hypotheses. The dependent variable was the SCL-SOM score and the independent variables were age, gender, medical disease status, psychiatric disease status, and the MEQ, CES-D, and STAI$S$ scores. As the associations between MEQ and SCL-SOM scores differed among those with/without medical disease and with/ without psychiatric disease, MEQ $\times$ medical disease status and

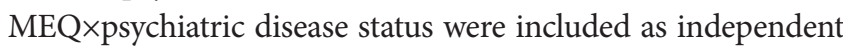
variables prior to performing additional multiple regressions. To define interactions, each interaction variable was individually included in all additional analyses.

\section{RESULTS}

\section{Characteristics of study subjects}

All subjects were divided into two groups by the median age of 40 years, as in a previous study [12]. A total of 78 subjects were $<40$ years in age, and 93 subjects were $\geq 40$ years in age. All subjects were also subgrouped by gender and by medical and psychiatric disease status. Forty-five subjects (26.3\%) had one or more medical diseases, and 44 (25.7\%) had one or more Axis I psychiatric disorders (Table 1). In terms of medical diseases, cardiovascular and musculoskeletal diseases were the most common (12 subjects). Ten subjects had endocrine diseases, six had ear-noseand-throat diseases, four had gynecological diseases, two had hepatic diseases, and two had neurological diseases. Two subjects had two or more medical diseases. In terms of psychiatric diseases, 24 subjects had depression (the most common disease), followed by anxiety in 14 . In addition, six subjects exhibited substancerelated disorders, one bipolar disorder, and two mental disorders caused by their general medical condition. Four subjects had two or more psychiatric diseases.

The mean MEQ score of all subjects was $50.4 \pm 7.8$. A positive correlation was evident between age and MEQ score $(r=0.223$, $\mathrm{p}=0.003$ ). The mean SCL-SOM and CES-D scores were 21.4 \pm 6.1 and $11.7 \pm 9.2$, respectively (Table 1 ). The SCL-SOM score was significantly associated with the CES-D and STAI-S scores (CES-D: $\mathrm{r}=0.354, \mathrm{p}<0.001$; STAI_S: $\mathrm{r}=0.311, \mathrm{p}<0.001)$. The SCL-SOM scores were significantly higher for subjects with medical or psychiatric diseases than for others (medical disease: $\mathrm{t}=-2.292, \mathrm{p}=0.023$; psy- 
Table 1. Comparison of demographic information, psychiatric comorbidity, morning-evening preference, somatization and depression between gender, age and disease groups

\begin{tabular}{|c|c|c|c|c|c|c|c|c|c|}
\hline & $\begin{array}{c}\text { Male } \\
(\mathrm{n}=73)\end{array}$ & $\begin{array}{l}\text { Female } \\
(\mathrm{n}=98)\end{array}$ & $\begin{array}{c}<40 \\
(\mathrm{n}=78)\end{array}$ & $\begin{array}{c}\geq 40 \\
(\mathrm{n}=93)\end{array}$ & $\begin{array}{c}\operatorname{Med}(+) \\
(n=45)\end{array}$ & $\begin{array}{l}\operatorname{Med}(-) \\
(\mathrm{n}=126)\end{array}$ & $\begin{array}{l}\text { Psy }(+) \\
(\mathrm{n}=44)\end{array}$ & $\begin{array}{c}\text { Psy (-) } \\
(\mathrm{n}=127)\end{array}$ & $\begin{array}{c}\text { Total } \\
(\mathrm{n}=171)\end{array}$ \\
\hline \multicolumn{10}{|l|}{ Demographic variables } \\
\hline Age (year)§ & $40.0 \pm 8.7$ & $41.8 \pm 7.8$ & $34.1 \pm 3.9^{\ddagger}$ & $46.9 \pm 6.0^{\ddagger}$ & $43.9 \pm 7.9^{*}$ & $40.0 \pm 8.1^{*}$ & $41.3 \pm 7.3$ & $41.0 \pm 8.5$ & $41.1 \pm 8.2$ \\
\hline Gender (male) $)^{\|}$ & - & - & $36(46.2)$ & $37(39.8)$ & $22(48.9)$ & $51(40.5)$ & $20(45.5)$ & $53(41.7)$ & $73(42.7)$ \\
\hline Medical disease"l & $22(30.1)$ & $23(23.5)$ & $14(17.9)^{*}$ & $31(33.3)^{*}$ & - & - & $15(34.1)$ & $30(23.6)$ & $45(26.3)$ \\
\hline Axis I. psychiatric disorder" & $20(27.4)$ & $24(24.5)$ & $20(25.6)$ & $24(25.8)$ & $15(33.3)$ & $29(23.0)$ & - & - & $44(25.7)$ \\
\hline \multicolumn{10}{|l|}{ Morningness-Eveningness } \\
\hline MEQ score§ & $48.7 \pm 8.9^{*}$ & $51.6 \pm 6.6^{*}$ & $49.5 \pm 8.1$ & $51.2 \pm 7.5$ & $50.5 \pm 8.9$ & $50.4 \pm 7.4$ & $49.7 \pm 6.3$ & $50.6 \pm 8.2$ & $50.4 \pm 7.8$ \\
\hline \multicolumn{10}{|l|}{ Somatization } \\
\hline SCL-SOM score§ & $22.0 \pm 6.2$ & $21.1 \pm 6.0$ & $21.2 \pm 6.1$ & $21.6 \pm 6.1$ & $23.2 \pm 6.5^{*}$ & $20.8 \pm 5.8^{*}$ & $23.1 \pm 6.4^{*}$ & $20.9 \pm 5.9^{*}$ & $21.4 \pm 6.1$ \\
\hline \multicolumn{10}{|l|}{ Depression and anxiety } \\
\hline CES-D score§ & $11.2 \pm 8.4$ & $12.0 \pm 9.8$ & $10.9 \pm 9.2$ & $12.3 \pm 9.2$ & $13.5 \pm 9.9$ & $11.0 \pm 9.0$ & $16.8 \pm 11.7^{\dagger}$ & $9.9 \pm 7.5^{\dagger}$ & $11.7 \pm 9.2$ \\
\hline STAI-S score ${ }^{\S}$ & $40.6 \pm 8.8$ & $40.0 \pm 10.0$ & $40.7 \pm 9.4$ & $39.9 \pm 9.6$ & $40.4 \pm 10.5$ & $40.2 \pm 9.1$ & $43.6 \pm 10.7^{*}$ & $39.1 \pm 8.8^{*}$ & $40.3 \pm 9.5$ \\
\hline
\end{tabular}

Data are expressed as mean \pm SD or $\mathrm{n}(\%) .{ }^{*} \mathrm{p}<0.05,{ }^{\dagger} \mathrm{p}<0.005,{ }^{\ddagger} \mathrm{p}<0.001$, \&results of independent $\mathrm{t}$-tests, "results of chi-square test. Med: Medical disease status, Psy: Psychiatric disease status, MEQ: Horne and Ostberg Morningness-Eveningness Questionnaire, SCL-SOM: Somatization subscale of the Symptom Checklist 90-item version, CES-D: Center for Epidemiological Studies-Depression Scale, STAI-S: The state anxiety subscale of Korean version of State Trait Anxiety Inventory

Table 2. Relationships between Somatization (SCL-SOM scale) and demographic variables (age, gender), morning-evening preference (MEQ score), medical disease status, psychiatric disease status, depressive symptoms (CES-D score), and anxiety symptoms (STAI-S) analyzed by multiple regression analysis

\begin{tabular}{|c|c|c|c|c|c|c|c|c|}
\hline & $\mathrm{R}^{2}$ & Age & Gender & MEQ & Med & Psy & CES-D & STAI-S \\
\hline Total $(n=171)$ & 0.182 & - & - & $\beta=-0.185^{\dagger}$ & - & - & $\beta=0.234^{\ddagger}$ & - \\
\hline Male $(\mathrm{n}=73)$ & 0.266 & - & - & $\beta=-0.186^{*}$ & - & - & $\beta=0.328^{\ddagger}$ & - \\
\hline Female $(\mathrm{n}=98)$ & 0.180 & - & - & $\beta=-0.201^{*}$ & $\beta=3.162^{*}$ & - & $\beta=0.164^{*}$ & - \\
\hline Age $<40(n=78)$ & 0.169 & - & - & $\beta=-0.216^{*}$ & - & - & $\beta=0.183^{*}$ & - \\
\hline Age $\geq 40(n=93)$ & 0.246 & - & - & $\beta=-0.184^{*}$ & $\beta=2.685^{*}$ & - & $\beta=0.261^{\ddagger}$ & - \\
\hline $\operatorname{Med}(+)(\mathrm{n}=45)$ & 0.122 & - & - & - & - & - & $\beta=0.230^{*}$ & - \\
\hline $\operatorname{Med}(-)(n=126)$ & 0.166 & - & - & $\beta=-0.179^{*}$ & - & - & $\beta=0.220^{\ddagger}$ & - \\
\hline Psy $(+)(n=44)$ & 0.260 & - & - & - & - & - & $\beta=0.277^{\ddagger}$ & - \\
\hline Psy (-) $(n=127)$ & 0.166 & - & - & $\beta=-0.243^{\ddagger}$ & - & - & $\beta=0.203^{\dagger}$ & - \\
\hline
\end{tabular}

${ }^{*} \mathrm{p}<0.05,{ }^{\dagger} \mathrm{p}<0.005,{ }^{\ddagger} \mathrm{p}<0.001$. Med: Medical disease status, Psy: Psychiatric disease status, MEQ: Horne and Ostberg Morningness-Eveningness Questionnaire, SCL-SOM: Somatization subscale of the Symptom Checklist 90-item version, CES-D: Center for Epidemiological Studies-Depression Scale, STAI-S: The state anxiety subscale of Korean version of State Trait

chiatric disease: $\mathrm{t}=-2.053, \mathrm{p}=0.042$ ). As expected, the CES-D score was significantly associated with psychiatric disease status $(\mathrm{t}=$ $-3.624, \mathrm{p}=0.001)$.

\section{The association between $\mathrm{M}-\mathrm{E}$ and somatization}

A significant Pearson correlation was evident between the MEQ and SCL-SOM scores $(\mathrm{r}=-0.235, \mathrm{p}=0.002)$. On multiple regression analysis, the MEQ and CES-D scores were independent determinants of the SCL-SOM score (MEQ: $\beta=-0.185, p=0.001$; CES-D: $\beta=0.234, p<0.001)$; somatization was thus independently influenced by M-E (Table 2).

\section{Characteristics by gender}

Of the 171 subjects, 73 were male and 98 female. The mean female MEQ score was significantly higher than that of males (males: $48.7 \pm 8.9$; females: $51.6 \pm 6.6, t=2.355, \mathrm{p}=0.020$ ). No other score or demographic datum differed significantly between males and fe- males (Table 1). Pearson analyses revealed significant correlations between the MEQ and SCL-SOM scores in both genders (males: $\mathrm{r}=-0.254, \mathrm{p}=0.030$; females: $\mathrm{r}=-0.201, \mathrm{p}=0.047$ ). Multiple regression analysis revealed that the MEQ and CES-D scores were independent determinants of the SCL-SOM scores for both genders (males: MEQ: $\beta=-0.186, p=0.011$; CES-D: $\beta=0.328, p<$ 0.001; females: MEQ: $\beta=-0.201, p=0.021$; CES-D: $\beta=0.164, p=$ $0.006)$. However, medical disease was an independent determinant of the SCL-SOM score only in females $(\beta=3.162, p=0.021)$ but not in males (Table 2).

\section{Characteristics by age}

A total of 78 subjects were $<40$ years in age and 93 were $\geq 40$ years in age. There was no significant difference between the two groups except in terms of age and medical disease status. As expected, a significantly greater number of older subjects reported medical diseases (chi-squared $=5.178, \mathrm{p}=0.023$ ). However, no sig- 
nificant age-related difference in psychiatric disease status was apparent (chi-squared $=0.001, \mathrm{p}=0.980$ ). The two age groups exhibited different relationships between their MEQ and SCL-SOM scores. A significant Pearson correlation was evident between the MEQ and SCL-SOM scores in younger $(\mathrm{r}=-0.306, \mathrm{p}=0.006)$ but not older $(\mathrm{r}=-0.181, \mathrm{p}=0.083)$ subjects. Multiple regression revealed that in those aged $<40$ years the MEQ and CES-D scores were independent determinants of the SCL-SOM score (MEQ: $\beta=-0.216$, $p=0.008$; CES-D: $\beta=0.183, p=0.011$ ), as was the case for all subjects. However, in those aged $\geq 40$ years, the MEQ and the CES-D scores, and medical disease satus were independent determinants of the SCL-SOM score (MEQ: $\beta=-0.184, p=0.017$; CES-D: $\beta=0.261, p<0.001$; medical disease: $\beta=2.685, p=0.026$ ) (Table 2).

\section{Characteristics by medical disease status}

Forty-five subjects had medical diseases and 126 did not. Apart from age and the SCL-SOM score, no significant difference was apparent between the two groups. The SCL-SOM score was significantly higher in those with medical diseases $(\mathrm{t}=-2.292, \mathrm{p}=0.023)$ (Table 1). Pearson correlation analysis showed that subjects lacking a medical disease exhibited a significant association between their MEQ and SCL-SOM scores, but those with medical diseases did not (medical disease: $\mathrm{r}=-0.266, \mathrm{p}=0.078$; no medical disease: $\mathrm{r}=-0.227, \mathrm{p}=0.010$ ). Similarly, in multiple regression analysis, the MEQ and CES-D scores were independent determinants of the SCL-SOM score in subjects without medical disease (MEQ: $\beta=$ $-0.179, p=0.007$; CES-D: $\beta=0.220, p<0.001$ ). Only the CES-D score (thus not the MEQ score) was an independent determinant of the SCL-SOM score in subjects with medical diseases (CES-D: $\beta=0.230, p=0.019$ ) (Table 2).

\section{Characteristics by psychiatric disease status}

Overall, 44 subjects had psychiatric diseases and 127 did not. As with medical disease status, psychiatric disease status was significantly associated with the SCL-SOM score ( $\mathrm{t}=-2.053, \mathrm{p}=0.042)$. The mean CES-D and STAI-S scores were also significantly higher in patients with psychiatric diseases (CES-D: $\mathrm{t}=-3.624$, $\mathrm{p}=$ 0.001; STAI-S: $\mathrm{t}=-2.736, \mathrm{p}=0.007$ ) (Table 1 ). The results varied by psychiatric disease status. No significant Pearson correlation was evident between the MEQ and SCL-SOM scores in subjects with psychiatric diseases $(r=0.068, p=0.661)$ but a significant correlation was apparent in those without psychiatric diseases $(r=-0.317$, $\mathrm{p}<0.001)$. Similarly, multiple regression analysis showed that the CES-D score was the only significant independent determinant of the SCL-SOM score in subjects with psychiatric diseases (CES-D: $\beta=0.277, p<0.001)$. Both the MEQ and CES-D scores were significant independent determinants of the SCL-SOM score in subjects without psychiatric disease (MEQ: $\beta=-0.243, p<0.001$; CES$\mathrm{D}: \beta=0.203, \mathrm{p}=0.002)$ (Table 2).

\section{Interactions between $\mathrm{M}-\mathrm{E}$ and medical or psychiatric disease status}

On multiple regression analysis exploring interactions among variables, MEQ scorexmedical disease status and MEQ scorex psychiatric disease status served as subgroup variables. There were no differences in other subgroups in additional analysis, but results were different from the initial analysis in female. In initial analyses, these variables had no effect in males, but showed significant effects in females. The MEQ score, medical disease status, and the CES-D score were significant independent determinants of the SCL-SOM score in primary analysis of females, but MEQ score $\times$ medical disease status (rather than the MEQ score alone) was a significant independent determinant upon additional analysis $\left(R^{2}=0.198\right.$, MEQ $\times$ medical disease status: $\beta=-0.199, p=0.006$; CES-D: $\beta=0.163, p=0.006$; medical disease status: $\beta=13.762$, $\mathrm{p}=0.001$ ).

\section{DISCUSSION}

We found that M-E predicted somatization independent of depression or anxiety status, and that the association was affected by medical and psychiatric disease status. The MEQ score independently predicted somatization in those without medical or psychiatric diseases, but not in those with diseases. Overall, in line with our first hypothesis, M-E and somatization were significantly associated, and the association was independent of depression and anxiety. Thus, M-E independently affects somatization. Evening types tended to exhibit more somatization, consistent with the results of previous studies, to the effect that evening types exhibited more psychiatric symptoms or disease than morning types $[3-5,8,9,13]$.

Several previous studies have shown that evening types are more susceptible to pain or pain-related diseases $[15,16,28,29]$. Pain sensitivity may vary among those with fibromyalgia or musculoskeletal disease [30,31]. This may be because the pain threshold is lower in evening types, although further studies are warranted. Evening types tended to have more psychiatric illnesses such as depression, perhaps enhancing somatization. Finally, morning and evening types differ in terms of personality trait, which may lead evening types to exhibit more somatization than morning types.

The association between M-E and somatization varied by medical or psychiatric disease status. M-E was associated with somatization in those lacking either disease type. The association between M-E and somatization was no more significant in those with medical diseases. This indicates that eveningness affects somatization, but less so when medical disease is present, suggesting that the somatic symptoms of patients with medical disease may be too serious to be affected by eveningness, given the much higher SCL-SOM scores of such subjects. Similarly, in those with psychiatric diseases, the association between M-E and somatization was not significant. It may be because the effect of M-E on somatization is relatively small due to both greater and more direct effect of psychiatric disorders on somatization.

Age and gender did not affect this association. Regardless of age or gender, evening types tended to exhibit more somatization, 
in contrast to existing studies reporting that the association between M-E and depression varied by age [5] and that the association between M-E and ADHD symptoms differed by gender [9]. This implies that the way M-E affects somatization is independent of depression or other psychiatric disease.

The interaction factor MEQ $\times$ medical disease status, but not the MEQ score alone, was an independent determinant of the SCLSOM score in females, which implies that M-E and medical diseases interacted; medical disease status was an independent determinant only in females, and the association between $\mathrm{M}-\mathrm{E}$ and somatization was affected by medical disease status. There are two possible reasons why the interaction was evident only in females. First, as the CES-D, STAI-S, and SCL-SOM scores did not differ significantly by gender, between-gender differences in personality traits (which we did not assess) may influence the interaction between MEQ scores and medical diseases more so than do psychiatric disorders. Second, the fact that more females than males were morning types may influence the interaction between MEQ score and medical disease status, although the mechanism remains unclear.

Associations between M-E and depression, and somatization and depression, have been well-studied. However, few studies have explored the relationship between M-E and somatization; no significant relationship was found by Putilov [17]. Several explanations of the difference in the results may be advanced. First, cultural factors may be in play. Culture affects both M-E [32-34] and somatization $[35,36]$. Second, most subjects evaluated by $\mathrm{Pu}-$ tilov [17] lived at high latitudes such as in Alaska or Western Siberia. Zeitgebers vary by latitude; M-E may thus affect psychiatric symptoms such as mood and fatigue differently at different latitudes. High latitudes in particular are affected by the polar night [37,38]. Third, Putilov [17] did not distinguish between those with and without medical and psychiatric diseases. We found that the association between M-E and somatization was significant only in subjects lacking disease. Finally, Putilov [17] used the SWPAQ to assess M-E, whereas we employed the MEQ.

Our finding that M-E and somatization were independently associated is meaningful because it contrasts with the findings of other studies. The principles or impact of influence will be able to be revealed on future research. However, our study had several limitations. First, the number of patients with medical or psychiatric diseases was small. However, the same result was obtained via multiple regression analysis when the number of determinants was reduced. Second, medical disease information was self-reported; thus, inaccuracies may have created bias. Third, our results cannot be readily generalized; most of our subjects were $20-50$ years in age and all were from the Incheon area. Additional follow-up studies with larger sample sizes and a wider geographic distribution are needed.

In conclusion, we found a significant association between M-E and somatization independent of any relationship with depression or anxiety; somatization was independently influenced by eveningness. The association was significant only in subjects without medical or psychiatric diseases.

\section{Acknowledgments}

This research was supported by the Brain Research Program through the National Research Foundation of Korea, funded by the Ministry of Science, ICT \& Future Planning (No. 2016M3C7A 1904336) and National Research Foundation of Korea (NRF) grant funded by the Korean government (MEST) (No. 2016 R1A2B4011561).

\section{Conflicts of Interest}

The authors have no potential conflicts of interest to disclose.

\section{Author Contributions}

Conceptualization: Seog Ju Kim, Seong-Jin Cho. Data curation: Seog Ju Kim, Seong-Jin Cho, Bum Joon Seok. Formal analysis: Sehyun Jeon, Bum Joon Seok. Funding acquisition: Seog Ju Kim. Investigation: Seog Ju Kim, Bum Joon Seok. Methodology: Seog Ju Kim, Seong-Jin Cho, Bum Joon Seok. Project administration: Seog Ju Kim. Resources: Seog Ju Kim, Seong-Jin Cho. Supervision: Seog Ju Kim. Software: Sehyun Jeon, Bum Joon Seok. Validation: Sehyun Jeon. Visualization: Bum Joon Seok. Writing-original draft: Bum Joon Seok. Writing_review \& editing: Sehyun Jeon, Seog Ju Kim.

\section{ORCID iDs}

Seog Ju Kim (1)

https://orcid.org/0000-0003-2467-5451

Bum Joon Seok (D)

https://orcid.org/0000-0001-9075-2609

\section{REFERENCES}

1. Busch FN. Clinical approaches to somatization. J Clin Psychol 2014;70:419427.

2. Horne JA, Ostberg O. A self-assessment questionnaire to determine morningness-eveningness in human circadian rhythms. Int J Chronobiol 1976;4: 97-110.

3. Chelminski I, Ferraro FR, Petros TV, Plaud JJ. An analysis of the "eveningness-morningness" dimension in "depressive" college students. J Affect Disord 1999;52:19-29.

4. Hidalgo MP, Caumo W, Posser M, Coccaro SB, Camozzato AL, Chaves ML. Relationship between depressive mood and chronotype in healthy subjects. Psychiatry Clin Neurosci 2009;63:283-290.

5. Kim SJ, Lee YJ, Kim H, Cho IH, Lee JY, Cho SJ. Age as a moderator of the association between depressive symptoms and morningness-eveningness. J Psychosom Res 2010;68:159-164.

6. Takeuchi H, Morisane H, Iwanaga A, Hino N, Matsuoka A, Harada T. Morningness-eveningness preference and mood in Japanese junior high school students. Psychiatry Clin Neurosci 2002;56:227-228.

7. Gau SS, Shang CY, Merikangas KR, Chiu YN, Soong WT, Cheng AT. Association between morningness-eveningness and behavioral/emotional problems among adolescents. J Biol Rhythms 2007;22:268-274.

8. Adan A. Chronotype and personality factors in the daily consumption of alcohol and psychostimulants. Addiction 1994;89:455-462.

9. Bae SM, Park JE, Lee YJ, Cho IH, Kim JH, Koh SH, et al. Gender difference in the association between adult attention deficit hyperactivity disorder symptoms and morningness-eveningness. Psychiatry Clin Neurosci 2010;64:649651.

10. Adan A, Lachica J, Caci H, Natale V. Circadian typology and temperament 
and character personality dimensions. Chronobiol Int 2010;27:181-193.

11. Caci H, Robert P, Boyer P. Novelty seekers and impulsive subjects are low in morningness. Eur Psychiatry 2004;19:79-84.

12. Lee S, Park JE, Cho SJ, Cho IH, Lee YJ, Kim SJ. Association between morningness-eveningness and temperament and character in community-dwelling Korean adults. Asia Pac Psychiatry 2014;6:77-82.

13. Park CI, An SK, Kim HW, Koh MJ, Namkoong K, Kang JI, et al. Relationships between chronotypes and affective temperaments in healthy young adults. J Affect Disord 2015;175:256-259.

14. Tonetti L, Fabbri M, Natale V. Relationship between circadian typology and big five personality domains. Chronobiol Int 2009;26:337-347.

15. Negriff S, Dorn LD. Morningness/eveningness and menstrual symptoms in adolescent females. J Psychosom Res 2009;67:169-172.

16. Takeuchi H, Oishi T, Harada T. Association between moringness-eveningness preference and mental/physical premenstrual symptoms in Japanese females 12 to 31 years of age. Chronobiol Int 2005;22:1055-1068.

17. Putilov AA. Associations of depression and seasonality with morning-evening preference: comparison of contributions of its morning and evening components. Psychiatry Res 2018;262:609-617.

18. Randler C. Age and gender differences in morningness-eveningness during adolescence. J Genet Psychol 2011;172:302-308.

19. Randler C, Freyth-Weber K, Rahafar A, Florez Jurado A, Kriegs JO. Morningness-eveningness in a large sample of German adolescents and adults. Heliyon 2016;2:e00200.

20. Knutson KL, von Schantz M. Associations between chronotype, morbidity and mortality in the UK Biobank cohort. Chronobiol Int 2018;35:1045-1053.

21. Basnet S, Merikanto I, Lahti T, Männistö S, Laatikainen T, Vartiainen E, et al. Associations of common noncommunicable medical conditions and chronic diseases with chronotype in a population-based health examination study. Chronobiol Int 2017;34:462-470.

22. Yu N, Wang S, Shin S. A study on the standardization of the Korean version of J.A Horne and O.Ostbergs' morningness-eveningness questionnaire and on the sleep pattern. J Korean Neuropsychiatr Assoc 1995;34:642-656.

23. Derogatis L, Cleary P. Confirmation of the dimensional structure of the SCL90: a study in construct validation. J Clin Psychol 1997;33:981-989.

24. Kim J, Kim G. The standardization study of symptom checklist-90-revision in Korea III. Ment Health Res 1984;2:278-311.

25. Radloff LS. The CES-D scale: a self-report depression scale for research in the general population. Appl Psychol Meas 1977;1:385-401.

26. Cho MJ, Kim KH. Use of the Center for Epidemiologic Studies Depression (CES-D) Scale in Korea. J Nerv Ment Dis 1998;186:304-310.

27. Hahn DW, Lee CH, Chon KK. Korean adaptation of Spielberger's STAI (KSTAI). Korean J Health Psychol 1996;1:1-14.

28. Bruni O, Russo PM, Ferri R, Novelli L, Galli F, Guidetti V. Relationships between headache and sleep in a non-clinical population of children and adolescents. Sleep Med 2008;9:542-548.

29. Jankowski KS. Morning types are less sensitive to pain than evening types all day long. Eur J Pain 2013;17:1068-1073.

30. Kantermann T, Theadom A, Roenneberg T, Cropley M. Fibromyalgia syndrome and chronotype: late chronotypes are more affected. J Biol Rhythms 2012;27:176-179.

31. Merikanto I, Lahti T, Seitsalo S, Kronholm E, Laatikainen T, Peltonen M, et al. Behavioral trait of morningness-eveningness in association with articular and spinal diseases in a population. PLoS One 2014;9:e114635.

32. Park YM, Matsumoto K, Seo YJ, Shinkoda H, Park KP. Scores on morningness-eveningness and sleep habits of Korean students, Japanese students, and Japanese workers. Percept Mot Skills 1997;85:143-154.

33. Randler C, Prokop P, Sahu S, Haldar P. Cross-cultural comparison of seven morningness and sleep-wake measures from Germany, India and Slovakia. Int J Psychol 2015;50:279-287.

34. Horzum MB, Randler C, Masal E, Beşoluk Ş, Önder İ, Vollmer C. Morningness-eveningness and the environment hypothesis - a cross-cultural comparison of Turkish and German adolescents. Chronobiol Int 2015;32:814-821.

35. Dreher A, Hahn E, Diefenbacher A, Nguyen MH, Böge K, Burian H, et al. Cultural differences in symptom representation for depression and somatization measured by the PHQ between Vietnamese and German psychiatric outpatients. J Psychosom Res 2017;102:71-77.

36. Ryder AG, Yang J, Zhu X, Yao S, Yi J, Heine SJ, et al. The cultural shaping of depression: somatic symptoms in China, psychological symptoms in North America? J Abnorm Psychol 2008;117:300-313.

37. Friborg O, Bjorvatn B, Amponsah B, Pallesen S. Associations between seasonal variations in day length (photoperiod), sleep timing, sleep quality and mood: a comparison between Ghana $\left(5^{\circ}\right)$ and Norway $\left(69^{\circ}\right)$. J Sleep Res 2012 21:176-184

38. Friborg O, Rosenvinge JH, Wynn R, Gradisar M. Sleep timing, chronotype, mood, and behavior at an Arctic latitude (69 N). Sleep Med 2014;15:798-807. 